\title{
3D FINITE ELEMENT ANALYSIS OF TUBE EXPANSION
}

\author{
M. A. Cavaliere ${ }^{1}$, Tomas Turkalj ${ }^{1}$, E. H. Giroldo ${ }^{2}$ \\ ${ }^{1}$ Tenaris Research \& Development, Argentina (mcavaliere@tenaris.com) \\ ${ }^{2}$ Tenaris Confab, Brazil
}

\begin{abstract}
Tube expansion is the last forming operation of the UOE process for the production of welded pipes. This process is carried out by pressing a steel plate into a $U$ and then $O$ shape prior to welding. Then a radial expansion is applied in order to obtain a final circumferential shape. The expander is composed of a truncated pyramid, which acts as a bar parallel to the tube axis, covered with several expanding tools (usually 8 to 12) which looks like truncated circular sectors. They are placed such that an axial displacement of the central bar produces the tube expansion. In order to improve the expander design, in particular to optimize the contact pressure distribution between the expanding tools and the central bar, a complex three dimensional numerical model of this process was developed considering frictional contact in the interactions between the different parts of the model (central bar, expanding tools and tube). Material non-linearity is also present since the tube is plastically deformed. Model results were useful to understand the impact of the expanding tool longitudinal profile on the contact pressure distribution and on the total horizontal force required to expand the tube.
\end{abstract}

Keywords: Finite element method, UOE process.

\section{INTRODUCTION}

In this work we present a 3D finite element analysis of the mechanical tube expansion process which is the last stage of the UOE process for producing longitudinally submerged arc welded (LSAW) tubes by cold forming plates. The first two letters of this process name stand for the shape of the plate after its main forming operations. As shown in Figure 1, a cylindrical punch forms the plate into a U-shape which is then press into an O-shape between two semicircular dies as shown in Figure. 2. It is important to explain that prior to these operations, the side borders of the plate are stamped with the final tube radius, to reduce the load in the O-press and improve shape. After welding the tube is expanded and a plastic deformation of $1 \%$ takes place in order to straighten and size the tubes.

In Figure 3 we show the complete layout of this process which is used to produce

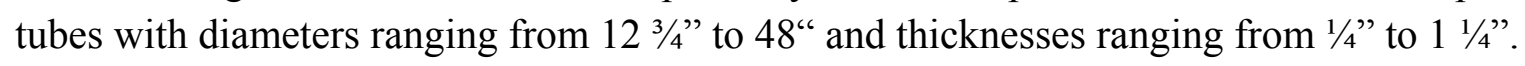




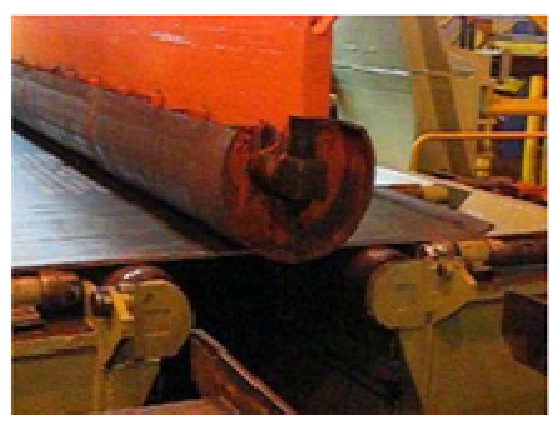

a) before pressing

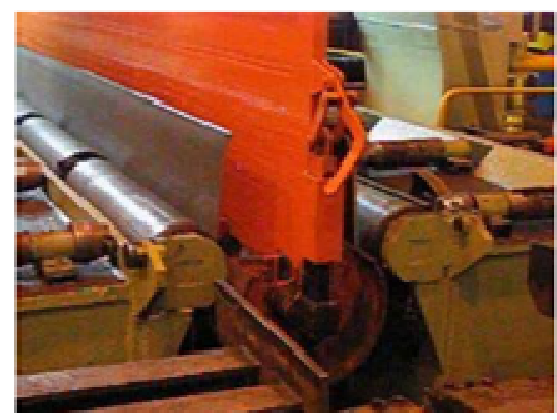

b) during pressing

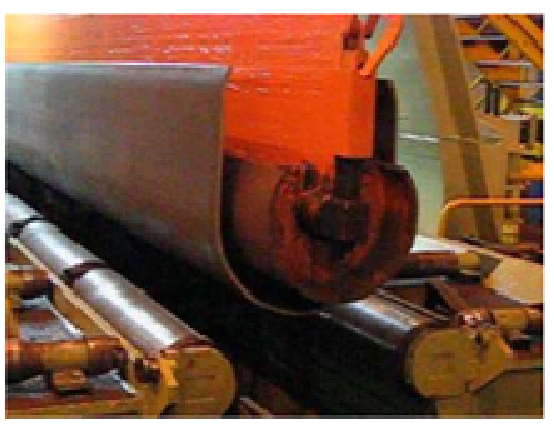

c) after pressing

Figure 1. Photographs of the plate shape at the U-press (Confab Industrial S.A.)

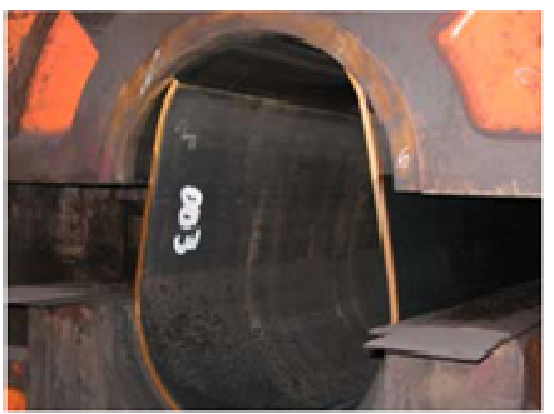

a) before pressing

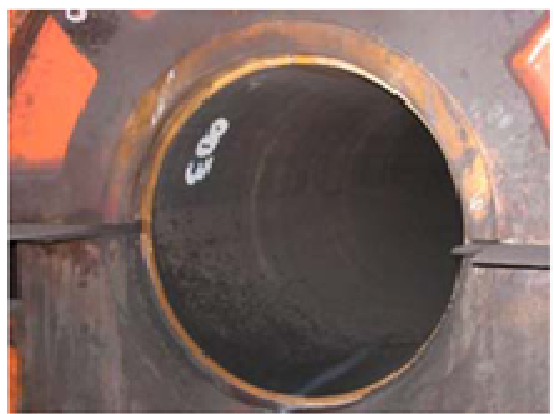

b) during pressing

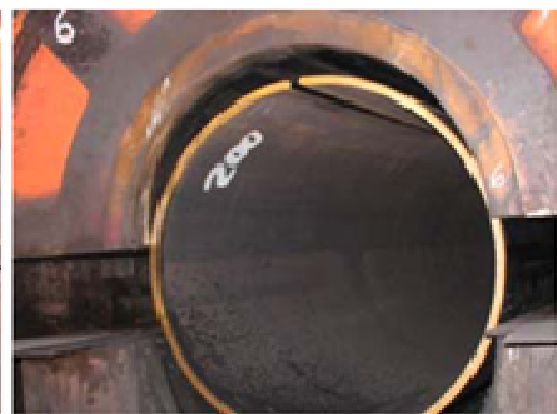

c) after pressing

Figure 2. Photographs of the plate shape at the O-press (Confab Industrial S.A.)

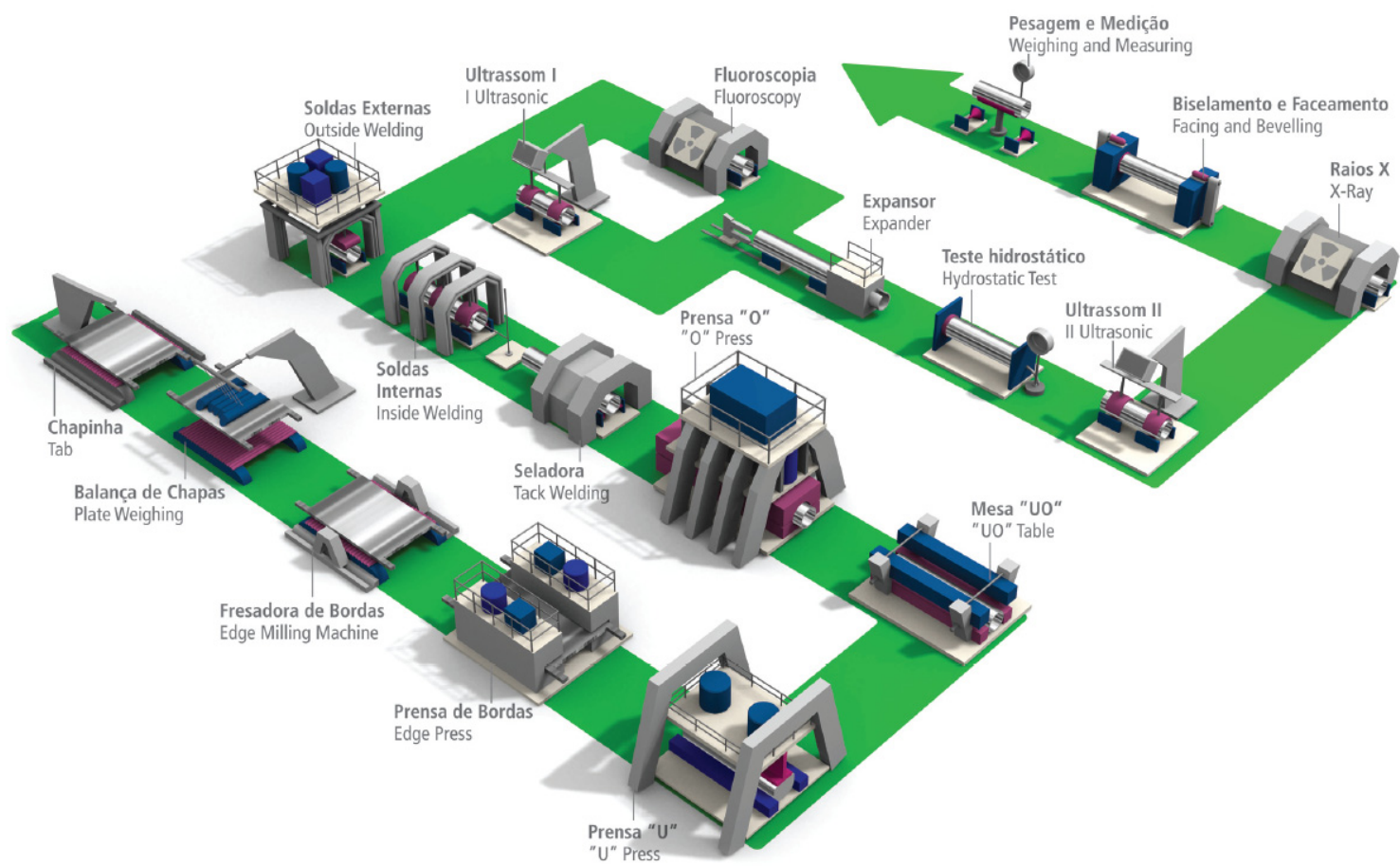

Figure 3. Layout of the UOE process for producing LSAW tubes. 
Several authors, including researchers from Tenaris, have developed 2D finite element models of the entire UOE forming process focused on the analysis of the process effects on the tube collapse pressure [1,2]. In this case it was necessary to develop a 3D model since the analysis is focused on the calculation of the contact pressure distribution between the internal tools that make up the expander equipment (shown in Figure 4),

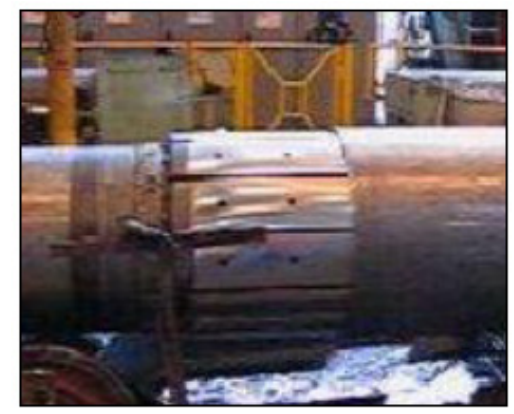

Figure 4. Photograph of the expander (Confab Industrial S.A.)

\section{FINITE ELEMENT MODEL DESCRIPTION}

The model includes the expander equipment and the tube as depicted in Figure 5. The initial position of the tube is shown in the figure, after expanding the first part of the tube, the simulation considers the tube movement to take into account the actual process.

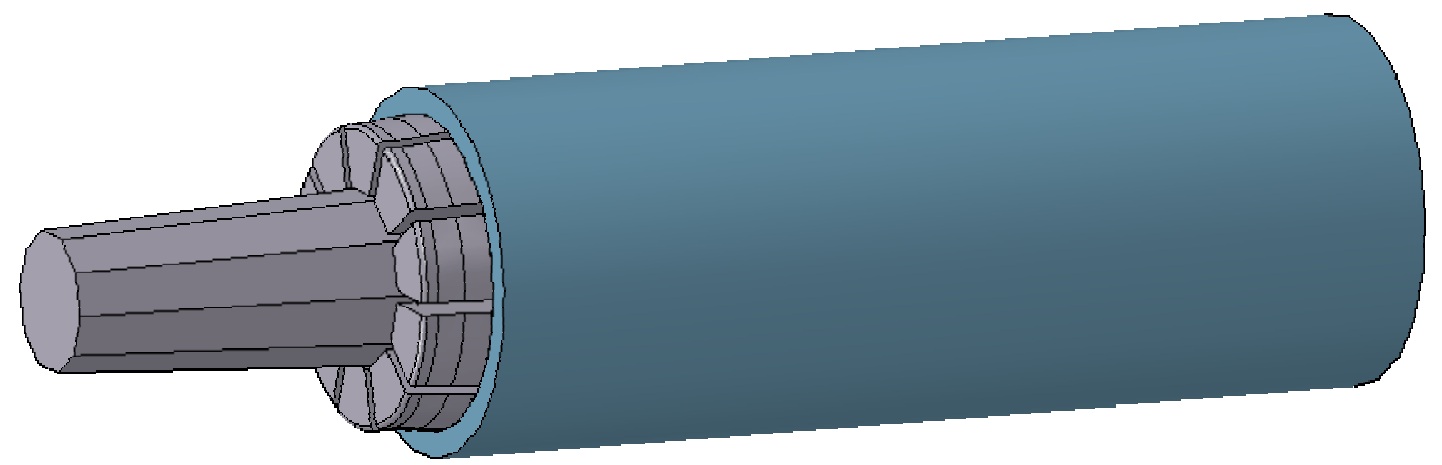

Figure 5. Expander and tube: initial position of the simulation.

The parts of the mechanical expander included in the model are shown at Figure 6. Two expanding segments were removed in that figure in order to visualize the internal parts. The expansion is produced by an axial force exerted over the central bar which pushes, in the shown case, 10 expanding segments in radial directions due to its taper. In this way the total expansion applied is controlled by the central bar movement. Oil lubricated bronze liners, attached to the inner surface of the expanding segments, slide over the central bar flat faces. In order to have an adequate lubrication, the normal contact pressure between those surfaces should be less than $150 \mathrm{MPa}$. 


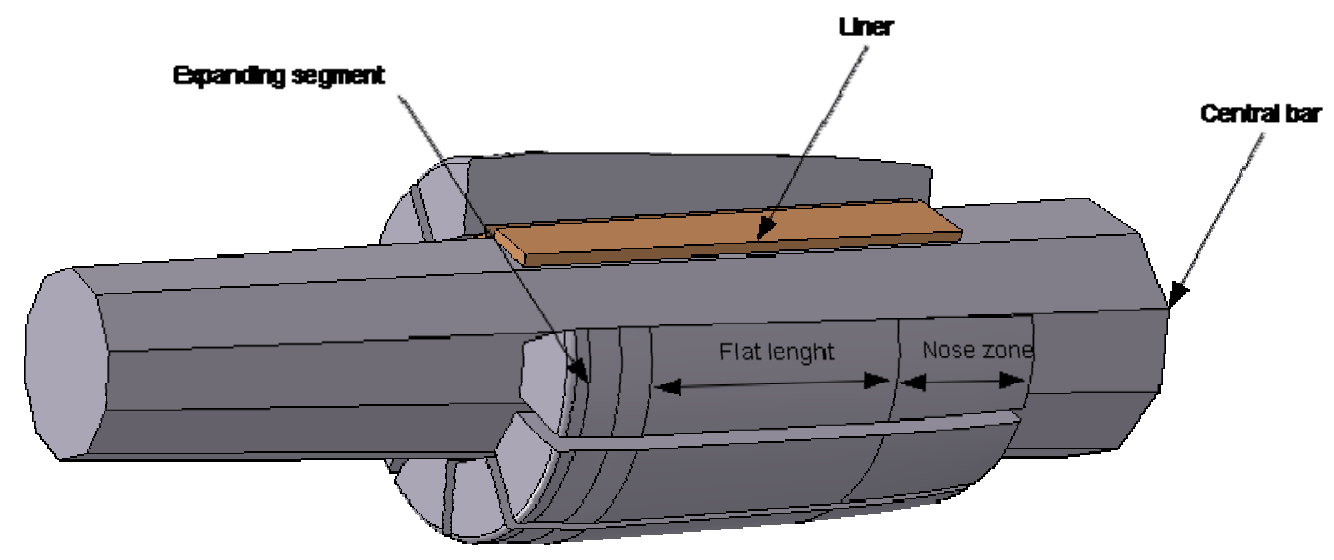

Figure 6. Perspective view of the expander parts included in the model.

The main object of this model is to calculate the distribution of the normal contact pressure between the liners and central bar because in some cases excessive wear was observed in the flat surfaces of the central bar. In accordance with that object, it is reasonable to neglect the weld seam and the residual stresses derived from the previous operations allowing the use of symmetries in order to model an $18^{\circ}$ sector, as shown in Figure 7.

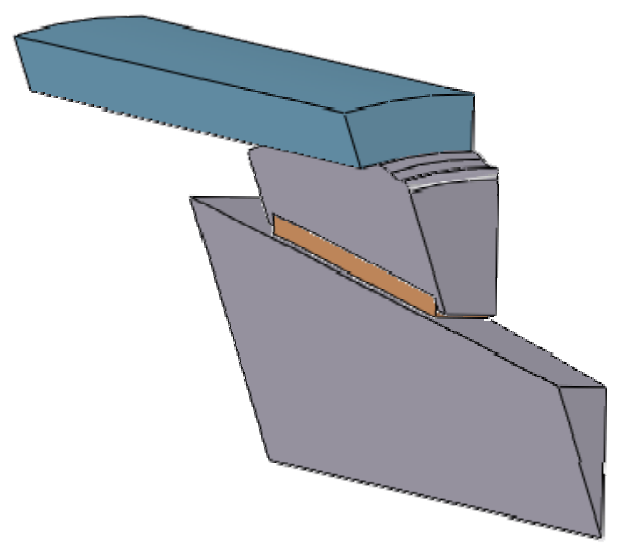

Figure 7. Perspective view of the $18^{\circ}$ model.

Although all the parametric analysis were performed using the $18^{\circ}$ model, we also developed a $90^{\circ}$ model, shown in Figure 8, in order to check the boundary conditions imposed in the $18^{\circ}$ model.

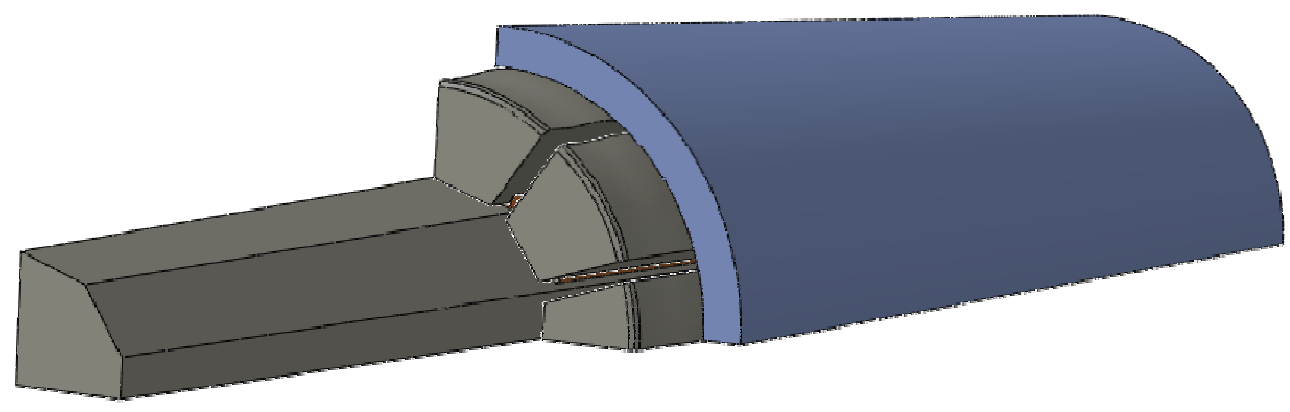

Figure 8 . Perspective view of the $90^{\circ}$ model. 
The computational power currently available enables the use of $3 \mathrm{D}$ solid elements in the entire model greatly simplifying the model development. If the tube is modeled using shell elements the computationally cost is reduced but it is necessary to deal with issues and difficulties derived from having contact between solid and shell elements. Using an adequate mesh refinement for meshing the tube (we use 8 linear elements across the thickness with an aspect ratio close to unity), it is possible to obtain an accurate distribution of deformation (across the tube thickness) and normal contact pressure (over the internal tube surface). For instance, the resulting finite element mesh for the $90^{\circ}$ model has 251.000 elements and was run using 8 cores in parallel in a workstation equipped with $32 \mathrm{~Gb}$. of RAM and 2 Quad Core Intel XEON $5550 @ 2.67 \mathrm{GHz}$ processors in an overnight run.

The tube material was modeled using Von Mises associated elasto-plastic constitutive relationship with a bilinear stress/strain relationship and isotropic hardening while the other materials (steel and bronze) were modeled as elastic material.

The major non-linearity of this problem is the contact interaction between the different parts of the mechanical expander and also between the tube and the expanding segments. In particular, special care is required at the contact interaction between the liner and the central bar because apart from considering Coulomb friction it is necessary to take into account the existence of finite sliding.

All this non-linearities were solved in an implicit way using Abaqus Standard.

\section{ANALYSIS OF MODEL RESULTS}

In this section we will show samples of the model results and explain it usefulness. The main result is the distribution of the normal contact pressure over the central bar faces, shown in Figure 9. The tube and one expanding sector in order were removed in order to show the result. It is important to remark that the contact between tube and expanding sector is not restricted to the flat zone as expected; in fact the major values are placed beyond of it.

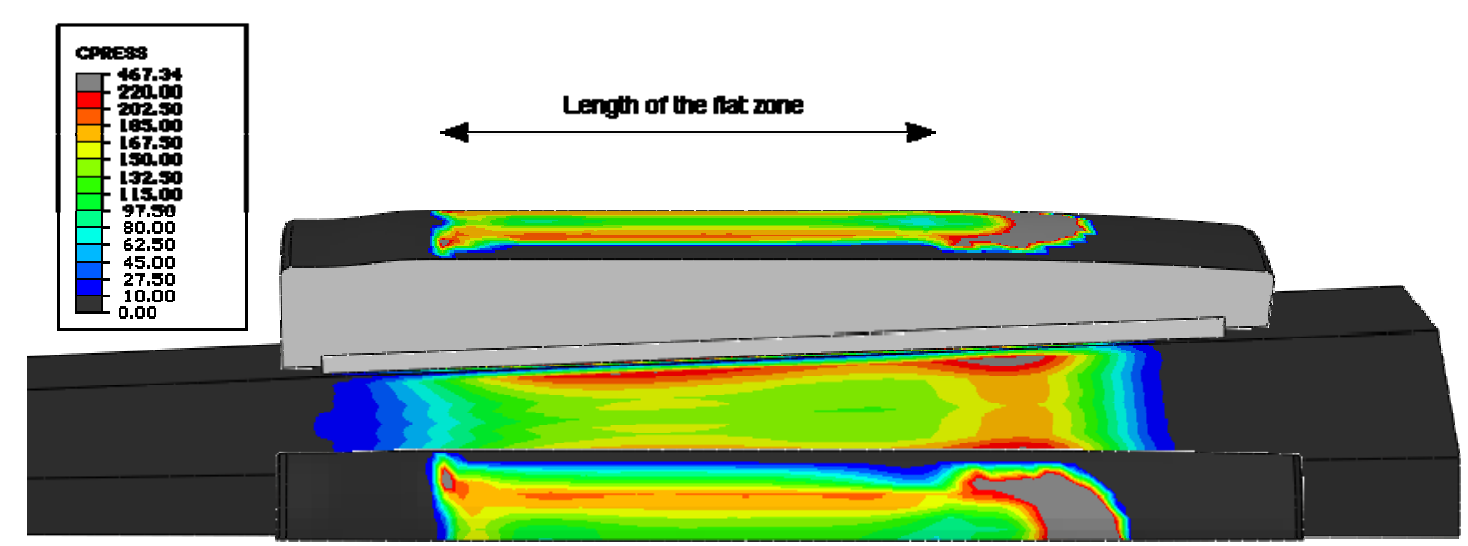

Figure 9. Contact pressures in MPa at the end of the expansion 
Another important result is the evolution of the total force required to push the central bar as a function of the permanent expansion applied, shown in Figure 10. It is important to remark that the model prediction of the force required to impose a small permanent expansion is similar to an analytical calculation of this force assuming a uniform hoop stress equal to yield stress and a uniform contact pressure distribution over the flat zone of the expanding sector. In the increment of pushing for with increasing expansion, is mainly explained by the increment of the contact zone between the tube and the expanding segments which is not taken into account in the analytical calculation.

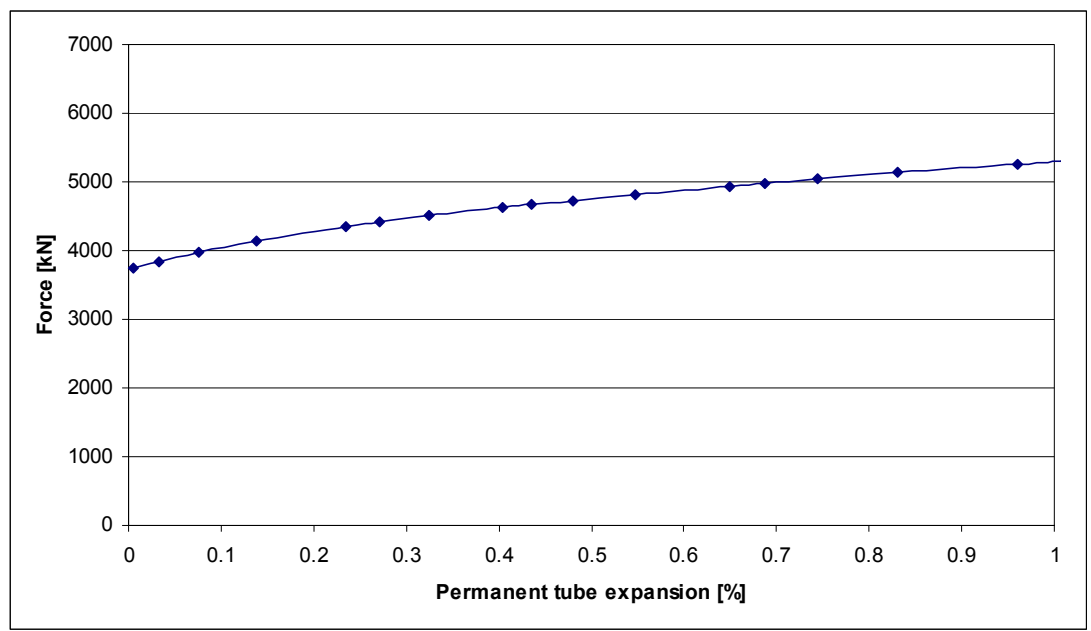

Figure 10. Evolution of the force to push the central bar during expansion

This model has been mainly used to analyze the effect of geometrical modifications of the expanding segment profile on the variables discussed in the previous paragraphs. It was also used to analyze the effect of modifying the pitch of tube advancement including its effect on the longitudinal profile of the expanded tube.

In addition, this model gives extra information about the effect of the expanding process on the tube useful for further analyses.

\section{Acknowledgements}

The support of Tenaris is greatly acknowledged.

\section{REFERENCES}

[1] Herynk M.D., Kyriakides S., Onoufriou A., Yun H.D., "Effects of the UOE/UOC pipe manufacturing processes on pipe collapse pressure". Int. J. of Mechanical Sciences 49, 533-558, 2007.

[2] Toscano R., Raffo J.L., Mantovano L., Fritz M., Silva R.C., "On the Influence of the UOE Process on Collapse and Collapse Propagation Pressure of Steel Deepwater Pipelines Under External Pressure", Offshore Technology Conference, OTC 18978, USA, May 2007. 